\title{
Risk classification of patients referred to secondary care for low back pain
}

\author{
Monica Unsgaard-Tøndel ${ }^{1,2^{*}}$, Ingunn Gunnes Kregnes ${ }^{3}$, Tom I. L. Nilsen ${ }^{2,4}$, Gunn Hege Marchand ${ }^{1,3}$ \\ and Torunn Askim ${ }^{1}$
}

\begin{abstract}
Background: Nonspecific low back pain is characterized by a wide range of possible triggering and conserving factors, and initial screening needs to scope widely with multilevel addressment of possible factors contributing to the pain experience. Screening tools for classification of patients have been developed to support clinicians. The primary aim of this study was to assess the criterion validity of STarT Back Screening Tool (STarT Back) against the more comprehensive Örebro Musculoskeletal Pain Questionnaire (ÖMPSQ), in a Norwegian sample of patients referred to secondary care for low back pain. Secondary aims were to assess risk classification of the patients, as indicated by both instruments, and to compare pain and work characteristics between patients in the different STarT Back risk categories.
\end{abstract}

Methods: An observational, cross-sectional survey among patients with low back pain referred to outpatient secondary care assessment at Trondheim University Hospital, Norway. Cohen's Kappa coefficient, Pearson's r and a Bland-Altman plot were used to assess criterion validity of STarT Back against ÖMPSQ. Furthermore, linear regression was used to estimate mean differences with 95\% Cl in pain and work related variables between the risk groups defined by the STarT Back tool.

Results: A total of 182 persons participated in the study. The Pearsons correlation coefficient for correspondence between scores on ÖMPSQ and STarT Back was 0.76. The Kappa value for classification agreement between the instruments was 0.35. Risk group classification according to STarT Back allocated 34.1\% of the patients in the low risk group, $42.3 \%$ in the medium risk, and $23.6 \%$ in the high risk group. According to ÖMPSQ, $24.7 \%$ of the participants were allocated in the low risk group, $28.6 \%$ in the medium risk, and $46.7 \%$ in the high risk group. Patients classified with high risk according to Start Back showed a higher score on pain and work related characteristics as measured by ÖMPSQ.

Conclusion: The correlation between score on the screening tools was good, while the classification agreement between the screening instruments was low. Screening for work factors may be important in patients referred to multidisciplinary management in secondary care.

Keywords: Primary care, Secondary care, Low back pain, Screening, Multidisciplinary, Work

\section{Background}

Low back pain is the leading cause of years lived with disability globally $[1,2]$. In addition to a negative impact on the individual's health, it is associated with substantial

\footnotetext{
* Correspondence: monica.unsgaard.tondel@ntnu.no

'Department of Neuromedicine and Movement Science (INB), NTNU, Faculty of Medicine and Health Sciences, N-7491 Trondheim, Norway

${ }^{2}$ Department of Public Health and Nursing, Norwegian University of Science and Technology, Faculty of Medicine and Health Sciences, Trondheim, Norway

Full list of author information is available at the end of the article
}

financial costs, partly for management that is not supported by scientific evidence [3].

Management guidelines suggest that patients seeking care for nonspecific and uncomplicated low back pain should be offered treatment in primary care [4]. On the other hand, patients with possible indicators of serious pathology or with compound treatment needs due to complex psychosocial challenges should be referred to specialist health service for further investigation and treatment [5]. Specific causes for low back pain are uncommon, and for about $85 \%$ of patients, low back pain

(c) The Author(s). 2018 Open Access This article is distributed under the terms of the Creative Commons Attribution 4.0 International License (http://creativecommons.org/licenses/by/4.0/), which permits unrestricted use, distribution, and reproduction in any medium, provided you give appropriate credit to the original author(s) and the source, provide a link to the Creative Commons license, and indicate if changes were made. The Creative Commons Public Domain Dedication waiver (http://creativecommons.org/publicdomain/zero/1.0/) applies to the data made available in this article, unless otherwise stated. 
is defined as nonspecific since the pain does not seem to be connected to specific organic impairments [6]. Nonspecific low back pain is characterized by a wide range of possible triggering and conserving factors, including lifestyle, behavioral, biomechanical, and psychosocial influences $[7,8]$. Therefore, initial screening needs to scope widely with multilevel addressment of possible factors contributing to the pain experience.

There are data suggesting that in general, previous management for patients with low back pain have failed to address its multifactorial nature and accordingly not contributed significantly to patients' improvement in the long term [8]. Part of the reason for this may be that multifactorial and knowledge based assessment tools have not been available. However, screening tools for classification of patients have been developed to support clinicians when identifying the specific needs of individual patients. The STarT Back screening tool (STarT Back) is one such screening tool [9]. It contains nine items covering eight domains, which were selected based on established prognostic factors suggested to affect probability of recovery. STarT Back was originally validated in England [9], and has been tested and adapted in a range of countries including Belgium [10], Denmark [11, 12], Finland [13], China [14], Germany [15], Norway [16] and Sweden [17]. Most of these studies have been performed in primary care. Another screening tool is the more comprehensive Örebro Musculoskeletal Pain Screening Questionnaire (ÖMPSQ), which was developed in Sweden for early identification of patients at risk for developing a persistent back problem [18]. ÖMPSQ has been considered an appropriate reference standard since it is an established tool to support clinicians in identifying patients in need of more comprehensive treatment for low back pain [19]. Since the performance of screening tools is highly context dependent, testing the tool in varied clinical settings is necessary. Therefore we wished to explore whether risk estimation by STarT Back is comparable to ÖMPSQ in a Norwegian multidisciplinary secondary care setting.

The primary aim of this study was to assess the criterion validity of the STarT Back Screening Tool against the Örebro Musculoskeletal Pain Screening Questionnaire in a Norwegian sample of patients with low back pain referred to assessment at a university hospital. Secondary aims were to assess risk classification of the patients, as indicated by both instruments, and to compare pain and work characteristics between patients in the different STarT Back risk categories.

\section{Methods}

\section{Design}

An observational, cross-sectional survey was performed in an outpatient multidisciplinary clinic for musculoskeletal pain in Trondheim University Hospital, Norway.

\section{Participants}

The study sample was patients referred for secondary line management because of low back pain. The inclusion criteria were as follows: Referred from their physician, manual therapist or chiropractor in the primary health care system with low back pain for more than 6 weeks. Exclusion criteria were age under 18, insufficient language capabilities, malignant disease, and unresolved social security or insurance problems.

\section{Variables}

\section{Background variables}

Background variables included age, gender, marital status, country of birth, educational level, and work status. Participants that were not out of work, sicklisted or on work assessment allowance were classified as employed. We defined people with any percentage of sickleave as sicklisted. Information on other diseases category was also collected, including headache, pulmonary disease, coronary disease, hypertension, diabetes and an open category for diseases specified by the participants.

\section{Measures}

STarT Back screening tool contains nine questions, and was developed based on prognostic factors for longstanding disability due to low back pain [9]. The questions cover the following eight constructs: bothersomeness, referred leg pain, comorbid pain, disability, catastrophizing, fear, anxiety, and depression. Based on dichotomizing responses patients were given an overall tool score and a psychosocial subscale score. Patients were allocated to the high risk group if the psychosocial subscale score was $\geq 4$, to the low risk group if the overall score was < 4 , and to the medium risk group if the overall score was $\geq 4$. Participants with missing items on STarT Back screening tool were excluded from the analysis if risk classification could not be established as described in the original study [9].

Örebro Musculoskeletal Pain Screening Questionnaire (ÖMPSQ) was developed to assist health care providers in assessing risk of developing a persistent back problem. Originally it was aiming at predicting risk for work absenteeism due to sickness [20]. The scoring system ranges from zero to 210, with higher scores indicating a higher risk of poor outcome. It has shown good psychometric properties $[18,21]$ and moderate predictive ability in identifying patients with spinal pain at risk of persisting pain and disability [22]. The questionnaire contains 25 items, and items 5-25 are scored [23]. Lower cut-off limits for ÖMPSQ were 89 for medium risk and 112 for high risk (corresponds to 42 and $53 \%$ of total score). Based on a recent study, we chose to omit the work questions in the ÖMPSQ total score for non-working patients [24]. Therefore the five work-related items no $6,8,16,17$, and 20) were excluded for participants out of work, and new 
scoring range and cut-off values were calculated based on the percentage of total score omitting five variables (i.e. 42 and 53\% of 160). This gave cutoffs of 67 and 85 for medium and high risk classification among non-workers, respectively.

\section{Analysis}

To assess criterion validity of STarT Back, the agreement in risk classification (low, medium, and high) based on STarT Back and ÖMPSQ was assessed by Cohen's Kappa coefficient. The calculations were done for the overall study population, but since some of the items of the ÔMPSQ are work related, we also estimated the agreement in risk classification for patients who were classified as workers. We also calculated the mean ÖMPSQ score with 95\% confidence interval (CI) according to STarT Back total score (Fig. 1). Since the two scores are measured on different scales, we converted both scores to percentage scores before we used Pearson's $r$ to estimate the correlation between STarT Back and ÖMPSQ. The acceptability limits were defined as: poor $\leq 30$; adequate $0.31-0.59$, and excellent $\geq 0.60$ [25]. The percentage scores were also used in a Bland-Altman plot assessing the agreement between STarT Back and ÖMPSQ screening tools.

Risk classification of the patients, as indicated by both instruments was described by a classification table. Finally, we used linear regression to estimate mean differences with $95 \% \mathrm{CI}$ in pain and work related variables between the three risk groups defined by the STarT Back tool. Non-normally distributed variables were analyzed using non-parametric Mann-Whitney $\mathrm{U}$ test and results presented as differences in median values between risk groups.

\section{Results}

A total of 300 patients received the study questionnaire, and 199 (66\%) returned it. After excluding 17 persons due to incomplete answers or age below 18 years, 182 patients (61\%) were available for statistical analysis. Among those, $73 \%$ were employed, mean age was 48 years $(\mathrm{SD}=15)$, and $51 \%$ were men (Table 1$)$.

The Kappa value for agreement between risk group classification was 0.35 (Table 2). Restricting the sample to only workers gave the same level of agreement (Kappa 0.36 ). Figure 1 shows that mean percentage of total ÖMPSQ-score increased monotonically with increasing STarT Back total score, and the two scores showed a high correlation $\left(r=0.76, \mathrm{p}^{<} 0.001\right)$. The Bland Altman plot (Fig. 2) display the difference between the two instruments according to the average percentage scores for both instruments, and suggests that the agreement between the instruments is highest for middle range scores. Mean bias is 1.6, but for patients scoring in the lower range on both scores there is tendency that STarT Back generates lower score than ÖMPSQ. On the other hand, in patients with higher average scores, STarT Back seems to generate higher scores than ÖMPSQ.

Start Back Screening tool allocated 34.1\% of the participants in the low risk group, $42.3 \%$ in the medium risk group, and $23.6 \%$ in the high risk group. Corresponding values for ÖMPSQ was $24.7 \%$ of the participants in the

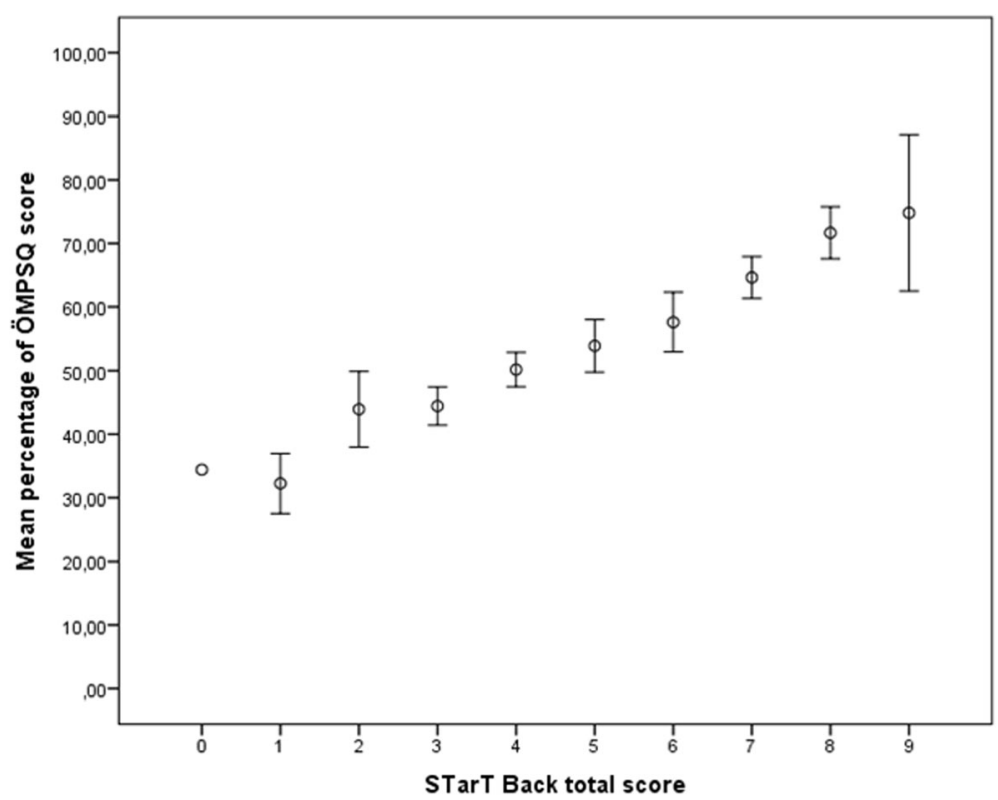

Fig. 1 Mean percent of Örebro Musculoskeletal Pain Score Questionnaire (ÖMPSQ) according to Start Back total score. Vertical bars represent 95\% confidence interval. (Pearson's $r=0.76$ ) 
Table 1 Characteristics of study population

\begin{tabular}{|c|c|c|c|c|c|c|c|}
\hline \multirow[t]{3}{*}{ Variables } & & & \multicolumn{5}{|c|}{ STarT Back risk groups } \\
\hline & \multicolumn{2}{|c|}{ Total } & \multirow{2}{*}{\multicolumn{2}{|c|}{$\frac{\text { Low }}{n=62}$}} & \multirow{2}{*}{\multicolumn{2}{|c|}{$\frac{\text { Medium }}{n=77}$}} & \multirow{2}{*}{$\frac{\text { High }}{n=43}$} \\
\hline & \multicolumn{2}{|c|}{$N=182$} & & & & & \\
\hline $\mathrm{Age}^{\mathrm{a}}$ & 48 & (15) & 47 & (15) & \multicolumn{2}{|c|}{$50 \quad(15)$} & $47 \quad(14)$ \\
\hline Gender, men ${ }^{b}$ & 92 & (51) & 29 & $(47)$ & 40 & $(52)$ & $23(54)$ \\
\hline Other diseases ${ }^{\mathrm{b}}$ & 127 & $(70)$ & 39 & $(63)$ & 57 & (74) & $31(72)$ \\
\hline Musculoskeletal comorbidity ${ }^{b}$ & 68 & (37) & 17 & (27) & 33 & (43) & $18(42)$ \\
\hline Married $^{b}$ & 97 & (53) & 30 & $(48)$ & 43 & (56) & $24(56)$ \\
\hline Born in Norway ${ }^{b}$ & 169 & (93) & 60 & $(97)$ & 73 & $(95)$ & $36(84)$ \\
\hline University / college education ${ }^{\text {b }}$ & 77 & $(42)$ & 31 & $(50)$ & 32 & $(42)$ & $14(33)$ \\
\hline Employed $^{\mathrm{b}}$ & 133 & (73) & 51 & (82) & 55 & (71) & $27 \quad(63)$ \\
\hline Sicklisted $^{\mathrm{b}}$ & 58 & (32) & 16 & $(26)$ & 26 & (34) & $16(37)$ \\
\hline
\end{tabular}

low risk group, $28.6 \%$ in the medium risk group, and $46.7 \%$ in the high risk group (Table 2). In supplementary analyses restricted to workers only, $38.3 \%$ were allocated in the low risk group, $41.4 \%$ in the medium risk group, and $20.3 \%$ in the high risk group as defined by STarT Back.

There was a dose-dependent relation between scores on pain and work items on ÖMPSQ and STarT Back risk group (Table 3), except for the item on job satisfaction. Pain variables differed most between low and medium risk group, while work variables separated most clearly between the medium and high risk group as defined by STarT Back.

\section{Discussion}

This study indicated high correlation between instrument scores and low agreement between risk classification between StarT Back tool and ÖMPSQ for patients referred to secondary care because of low back pain. Risk group classification according to STarT Back allocated $23.6 \%$ in the high risk group. According to ÖMPSQ, $46.7 \%$ were allocated in the high risk group. Patients classified with high risk according to Start Back showed a higher score on pain and work related characteristics as measured by ÖMPSQ.
The Start Back total score highly correlated with ÖMPSQ total score, indicating good criterion validity for STarT Back in a Norwegian sample of low back pain patients referred to a multidisciplinary outpatient clinic in secondary care. This result is comparable with previous studies performed in other countries and settings. Bruyere and coworkers [10] addressed the correlation between the ÖMPSQ and STarT Back and found a Spearman correlation coefficient of 0.74 . The latter study included patients in settings different from the present study; a rehabilitation center, a back school, a private physiotherapy unit as well as persons with low back pain at a fitness center [10]. ÖMPSQ has also been compared to STarT Back screening tool in England, including two hundred and forty-four consecutive non-specific low back pain consulters at general practitioners [19]. They found a correlation between STarT Back tool and ÖMPSQ of 0.80 and classification agreement Kappa 0.57. Significant differences between STarT Back and ÖMPSQ-registered threshold were observed in that STarT Back allocated fewer patients to high risk classification.

Despite very good correlation between the two scales, the findings from the risk classification in the present study showed that 22 out of the 52 patients classified as medium risk by ÖMPSQ were classified as low risk on Start Back. Additionally, 41 out of the 85 patients with high risk according to ÖMPSQ were classified with moderate risk according to Start Back, in line with the study from England [19]. One may ask whether these discrepancies were related to the fact that ÖMPSQ has five work-related questions and have been suggested to be a good predictor of future absenteeism [18], while Start Back does not include any work questions. Studies assessing the validity of the short-form ÖMPSQ that includes ten items, of which two covers work that is optional connected to the home or to paid work may support this hypothesis as it showed less discrepancies in classification when compared to STarT Back [17, 26]. Because STarT Back does not cover work, we hypothesized that STarT Back could underestimate risk for participants with work-related obstacles for recovery. Our results showed that patients classified with high risk

Table 2 Classification table showing agreement between risk group stratification as defined by STarT Back and Örebro screening tools

\begin{tabular}{|c|c|c|c|c|c|}
\hline \multirow[b]{2}{*}{ Start Back Screening tool risk group } & \multicolumn{5}{|c|}{ Örebro Musculoskeletal Pain Questionnaire risk group } \\
\hline & Low & Medium & High & Total & Percentage \\
\hline Low & 35 & 22 & 5 & 62 & 34.1 \\
\hline Medium & 9 & 27 & 41 & 77 & 42.3 \\
\hline High & 1 & 3 & 39 & 43 & 23.6 \\
\hline Total & 45 & 52 & 85 & 182 & \\
\hline Percentage & 24.7 & 28.6 & 46.7 & & \\
\hline
\end{tabular}




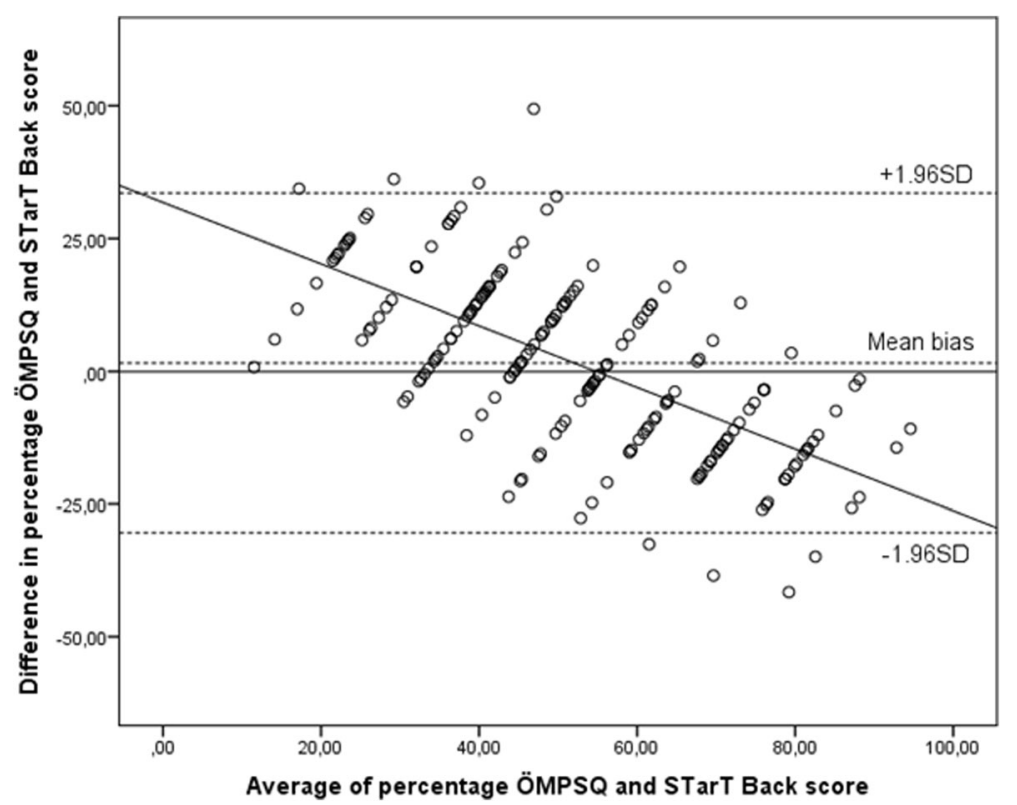

Fig. 2 Agreement between STarT Back screening tool and Örebro Musculoskeletal Pain Screening Questionnaire (ÖMPSQ). Dotted lines in the Bland-Altman plot represent mean bias with 95\% limits of agreement. The solid diagonal line represent the difference between the screening tools regressed on the average of the two tools (slope $=-0.58$ ). X-axis displays average percentage score between both instruments. Y-axis display differences in percentage score

according to Start Back showed a significantly higher score on work related characteristics as measured by ÖMPSQ, with one exception for the item on job satisfaction. These findings suggest that specifically screening for work factors is important in this group of patients. This is also indicated by the difference in ÖMPSQ-scores between workers and non-workers, in line with results from a recent factors analysis [27]. Further research is needed to confirm this relation, and to address the need for more knowledge regarding referral practice and the right candidates for multidisciplinary rehabilitation to restore employability [28].

Another finding was that the agreement for score was best for middle range scores, and that the compliance between tools were lower in both ends of the scoring scales. For the higher mean percentage scores, the tendency was that STarT Back tool allocated higher score. In spite of that, Örebro allocated a higher percentage of participants to the high risk group, again indicating a lack of correspondence between the cut-off limits for the instruments.

The results indicated that between 24.7 (ÖMPSQ) and 34.1 (STarT Back) percent of the respondents had low risk for longstanding disability due to low back pain. Clinical guidelines recommend secondary care referral when management needs are too complex for primary care management [4]. Given that, it is somewhat surprising that as much as $25-34 \%$ of the patients were classified low risk. Multidisciplinary management in secondary care is the recommended treatment choice for patients with significant obstacles to recovery and / or when previous treatment have not been effective [5]. In general, secondary care referral may be due to complex psychosocially oriented treatment needs or to suspicion of organic disease from the primary care contact. The outpatient clinic in the present study offers multidisciplinary treatment targeting psychosocial needs as well as examination of potential pathoanatomic triggers. Therefore, screening tools designed for early addressing of psychosocial obstacles for recovery in primary care may not be sufficient or relevant to consider if a patient should be managed in secondary care.

To our knowledge, no studies have compared scores and classification by these two screening instruments in patients referred to secondary care. On the other hand, the predictive value of both instruments in secondary care has been evaluated. An Australian study concluded that the instruments add no further value over and above clinical judgement [29], and a Danish study concluded that the predictive ability of STarT Back is less good in secondary care compared to primary care [30]. Again, it is plausible to suggest that the most relevant screening items for primary and secondary care patients differ.

\section{Strengths and limitations}

This is a cross-sectional study, and it cannot evaluate the predictive value of the screening instruments. The 
Table 3 Mean differences from linear regression analyses ${ }^{a}$ of selected pain and work related variables between Start Back risk groups (work related variables analyzed only on people in work, and higher scores represent worse problems)

\begin{tabular}{llll}
\hline & \multicolumn{3}{l}{ STarT Back risk group } \\
\cline { 2 - 4 } & Low risk & Medium risk & High risk \\
\hline Pain & & & \\
Intensity last week, 1-10 points & & \\
Mean (SD) & $3.8(2.0)$ & $5.1(2.5)$ & $5.7(2.7)$ \\
Difference (95\% Cl) & 0.0 (reference) & $1.2(0.3-2.1)$ & $1.9(0.8-2.9)$ \\
Intensity past 3 months, 1-10 points & & \\
Mean (SD) & $6.0(1.5)$ & $6.9(1.5)$ & $7.9(1.5)$ \\
Difference (95\% Cl) & 0.0 (reference) & $1.0(0.4-1.5)$ & $1.9(1.2-2.6)$ \\
Duration, weeks & & & \\
Median (IQR) & 46 (20-52) & $52(33-52)$ & $52(47-52)$ \\
Difference ( $p$-value) & 0 (reference) & $8(0.007)$ & $8(0.004)$
\end{tabular}

Work

Missed days of work, passed 18 months

$\begin{array}{llll}\text { Median (IQR) } & 11(0-137) & 30(5-137) & 137(60-365) \\ \text { Difference ( } p \text {-value) } & 0 \text { (reference) } & 19(0.14) & 126(0.001)\end{array}$

Heavy / monotoneous work, 1-10 points

$\begin{array}{llll}\text { Median (IQR) } & 5(3-7) & 7(5-8) & 8(6-9) \\ \text { Difference (p-value) } & 0 \text { (reference) } & 2(0.02) & 3(0.004)\end{array}$

Workable in six months, 1-10 points

$\begin{array}{llll}\text { Median (IQR) } & 1(0-5) & 2(0-5) & 6(2-9) \\ \text { Difference (p-value) } & 0 \text { (reference) } & 1(0.13) & 5(<0.001)\end{array}$

Job satisfaction, 1-10 points

$\begin{array}{llll}\text { Median (IQR) } & 2(1-4) & 2(1-5) & 3(1-5) \\ \text { Difference (p-value) } & 0 \text { (reference) } & 0(0.23) & 1(0.17)\end{array}$

Should not work (fear), 1-10 points

$\begin{array}{llll}\text { Median (IQR) } & 4(0-6) & 5(3-9) & 10(6-10) \\ \text { Difference (p-value) } & 0 \text { (reference) } & 1(0.01) & 6(<0.001)\end{array}$

${ }^{a}$ Non-normally distributed variables were analyzed using non-parametric Mann-Whitney $\mathrm{U}$ test and results presented as differences in median values between risk groups

response rate was $66 \%$ and we cannot rule out that the study participants were different from the non-responders. Consequently, the results may not automatically be generalisable to the population of patients referred to the multidisciplinary clinic. Missing responses on some items may have introduced bias. To compensate for missing on the work items, supplementary analyses were performed to compare whole-sample results to results from analyses restricted to patients in work.

The results from the present study do not suggest that the risk classification by STarT Back is comparable to risk classification by ÖMPSQ for patients referred to secondary care for low back pain, though the instruments' scores correlated well. This study also suggests that around one third of the patients referred to secondary care will be classified low risk according to these primary care screening tools. The results do not support the applicability of StarT Back screening tool as decision support in Norwegian secondary health care. This may be due to differences in timing of testing, clinical setting, and study sample compared to the original target group for screening. The results also indicated that the working items in ÖMPSQ may be central when addressing psychosocial load in working patients.

\section{Conclusion}

STarT Back scores correlated well to scores on ÖMPSQ, while classification agreement between the instruments was low in patients referred to multidisiplinary secondary care for low back pain. Patients classified as high risk by STarT Back reported more challenges connected to work on ÖMPSQ sub-items.

\section{Abbreviations \\ Cl: Confidence interval; ÖMPSQ: Örebro Musculoskeletal Pain Screening Questionnaire; STarT Back: STarT Back screening tool}

\section{Acknowledgements}

The authors would like to thank the study participants for their contribution in the data collection.

\section{Availability of data and materials}

The participant included in the present study did not consent to sharing of the raw data. Hence, due to Norwegian regulations and conditions for informed consent, the dataset is not publicly available.

\section{Authors' contributions}

IGK, MUT and TA designed the study. TILN and MUT analyzed the data. MUT wrote the paper. GHM, IGK, MUT, TA and TILN discussed the results, commented on the manuscript, and approved the final version.

Ethics approval and consent to participate

The Regional Committee for Medical and Health Research Ethics in NorthNorway (REC North) defined the project as health service research. Suchresearch is not regulated by the Norwegian legislation for health research(2014/341/REC North). Hence, the project was approved by the NorwegianCentre for Research Data (No 38883). Informed consent was signed by allparticipants.

\section{Competing interests}

The authors declare that they have no competing interests.

\section{Publisher's Note}

Springer Nature remains neutral with regard to jurisdictional claims in published maps and institutional affiliations.

\section{Author details}

${ }^{1}$ Department of Neuromedicine and Movement Science (INB), NTNU, Faculty of Medicine and Health Sciences, N-7491 Trondheim, Norway. ${ }^{2}$ Department of Public Health and Nursing, Norwegian University of Science and Technology, Faculty of Medicine and Health Sciences, Trondheim, Norway. ${ }^{3}$ Department of Physical Medicine and Rehabilitation, St. Olav's Hospital, Trondheim University Hospital, Trondheim, Norway. ${ }^{4}$ Clinic of Anaesthesia and Intensive Care, St Olavs Hospital, Trondheim University Hospital,

Trondheim, Norway. 
Received: 20 December 2017 Accepted: 8 May 2018

Published online: 24 May 2018

\section{References}

1. Hoy DG, Smith E, Cross M, Sanchez-Riera L, Blyth FM, Buchbinder R, Woolf AD, Driscoll T, Brooks P, March LM. Reflecting on the global burden of musculoskeletal conditions: lessons learnt from the global burden of disease 2010 study and the next steps forward. Ann Rheum Dis. 2015;74(1):4-7.

2. March L, Smith EU, Hoy DG, Cross MJ, Sanchez-Riera L, Blyth F, Buchbinder R, Vos T, Woolf AD. Burden of disability due to musculoskeletal (MSK) disorders. Best Pract Res Clin Rheumatol. 2014;28(3):353-66.

3. Srinivas SV, Deyo RA, Berger ZD. Application of "less is more" to low back pain. Arch Intern Med. 2012;172(13):1016-20.

4. Koes BW, van Tulder M, Lin CW, Macedo LG, McAuley J, Maher C. An updated overview of clinical guidelines for the management of non-specific low back pain in primary care. Eur Spine J. 2010;19(12):2075-94.

5. Bernstein IA, Malik Q, Carville S, Ward S. Low back pain and sciatica: summary of NICE guidance. BMJ. 2017;356:16748.

6. van Tulder M, Becker A, Bekkering T, Breen A, del Real MT, Hutchinson A Koes B, Laerum E, Malmivaara A, Care CBWGoGftMoALBPiP. Chapter 3. European guidelines for the management of acute nonspecific low back pain in primary care. Eur Spine J. 2006;15 Suppl 2:S169-91.

7. Christensen J, Fisker A, Mortensen EL, Olsen LR, Mortensen OS, Hartvigsen J, Langberg $\mathrm{H}$. Comparison of mental distress in patients with low back pain and a population-based control group measured by symptoms check list-a case-referent study. Scand J Public Health. 2015;43(6):638-47.

8. O'Sullivan P, Caneiro JP, O'Keeffe M, O'Sullivan K. Unraveling the complexity of low back pain. J Orthop Sports Phys Ther. 2016;46(11):932-7.

9. Hill JC, Dunn KM, Lewis M, Mullis R, Main CJ, Foster NE, Hay EM. A primary care back pain screening tool: identifying patient subgroups for initial treatment. Arthritis Rheum. 2008;59(5):632-41.

10. Bruyere O, Demoulin M, Beaudart C, Hill JC, Maquet D, Genevay S, Mahieu G, Reginster JY, Crielaard JM, Demoulin C. Validity and reliability of the French version of the STarT back screening tool for patients with low back pain. Spine (Phila Pa 1976). 2014;39(2):E123-8.

11. Morso L, Albert H, Kent P, Manniche C, Hill J. Translation and discriminative validation of the STarT back screening tool into Danish. Eur Spine J. 2011; 20(12):2166-73.

12. Morso L, Kent P, Albert HB, Hill JC, Kongsted A, Manniche C. The predictive and external validity of the STarT back tool in Danish primary care. Eur Spine J. 2013;22(8):1859-67.

13. Piironen S, Paananen M, Haapea M, Hupli M, Zitting P, Ryynanen K, Takala EP, Korniloff K, Hill JC, Hakkinen A, et al. Transcultural adaption and psychometric properties of the STarT back screening tool among Finnish low back pain patients. Eur Spine J. 2016;25(1):287-95.

14. Luan S, Min Y, Li G, Lin C, Li X, Wu S, Ma C, Hill JC. Cross-cultural adaptation, reliability, and validity of the Chinese version of the STarT back screening tool in patients with low back pain. Spine (Phila Pa 1976). 2014:39(16):E974-9.

15. Karstens S, Krug K, Hill JC, Stock C, Steinhaeuser J, Szecsenyi J, Joos S. Validation of the German version of the STarT-back tool (STarT-G): a cohort study with patients from primary care practices. BMC Musculoskelet Disord. 2015;16:346

16. Robinson HS, Dagfinrud H. Reliability and screening ability of the StarT back screening tool in patients with low back pain in physiotherapy practice, a cohort study. BMC Musculoskelet Disord. 2017;18(1):232.

17. Forsbrand M, Grahn B, Hill JC, Petersson IF, Sennehed CP, Stigmar K. Comparison of the Swedish STarT back screening tool and the short form of the Orebro musculoskeletal pain screening questionnaire in patients with acute or subacute back and neck pain. BMC Musculoskelet Disord. 2017: 18(1):89.

18. Linton SJ, Boersma K. Early identification of patients at risk of developing a persistent back problem: the predictive validity of the Orebro musculoskeletal pain questionnaire. Clin J Pain. 2003;19(2):80-6.

19. Hill JC, Dunn KM, Main CJ, Hay EM. Subgrouping low back pain: a comparison of the STarT back tool with the Orebro musculoskeletal pain screening questionnaire. Eur J Pain. 2010;14(1):83-9.

20. Linton SJ, Hallden K. Can we screen for problematic back pain? A screening questionnaire for predicting outcome in acute and subacute back pain. Clin J Pain. 1998;14(3):209-15.
21. Maher CG, Grotle M. Evaluation of the predictive validity of the Orebro musculoskeletal pain screening questionnaire. Clin J Pain. 2009;25(8):666-70.

22. Hockings RL, McAuley JH, Maher CG. A systematic review of the predictive ability of the Orebro musculoskeletal pain questionnaire. Spine (Phila $\mathrm{Pa}$ 1976). 2008;33(15):E494-500.

23. Ruokolainen O, Haapea M, Linton S, Korniloff K, Hakkinen A, Paananen M, Karppinen J. Construct validity and reliability of Finnish version of Orebro musculoskeletal pain screening questionnaire. Scand J Pain. 2016;13:148-53.

24. Soer R, Vroomen P, Stewart R, Coppes M, Stegeman P, Dijkstra P, Reneman M. Factor analyses for the Orebro musculoskeletal pain questionnaire for working and nonworking patients with chronic low back pain. Spine J. 2017;17(4):603-9.

25. Andresen EM. Criteria for assessing the tools of disability outcomes research. Arch Phys Med Rehabil. 2000;81(12 Suppl 2):S15-20.

26. Fuhro FF, Fagundes FR, Manzoni AC, Costa LO, Cabral CM. Orebro musculoskeletal pain screening questionnaire - short form and Start back screening tool: correlation and agreement analysis. Spine (Phila Pa 1976). 2016;41(15):E931-6.

27. Soegaard R, Christensen FB, Christiansen T, Bunger C. Costs and effects in lumbar spinal fusion. A follow-up study in 136 consecutive patients with chronic low back pain. Eur Spine J. 2007;16(5):657-68.

28. Pedersen $\mathrm{P}$, Nielsen CV, Jensen OK, Jensen C, Labriola M. Employment status five years after a randomised controlled trial comparing multidisciplinary and brief intervention in employees on sick leave due to low back pain. Scand J Public Health. 2018;46(3):383-8.

29. Karran EL, Traeger AC, McAuley JH, Hillier SL, Yau YH, Moseley GL. The value of prognostic screening for patients with low back pain in secondary care. J Pain. 2017;18(6):673-86.

30. Morso L, Kent P, Manniche C, Albert HB. The predictive ability of the STarT back screening tool in a Danish secondary care setting. Eur Spine J. 2014; 23(1):120-8.

\section{Ready to submit your research? Choose BMC and benefit from:}

- fast, convenient online submission

- thorough peer review by experienced researchers in your field

- rapid publication on acceptance

- support for research data, including large and complex data types

- gold Open Access which fosters wider collaboration and increased citations

- maximum visibility for your research: over $100 \mathrm{M}$ website views per year

At BMC, research is always in progress.

Learn more biomedcentral.com/submissions 